\title{
Identification of Significant Prognostic Tissue Markers Associated with Survival in Upper Urinary Tract Urothelial Carcinoma Patients Treated with Radical Nephroureterectomy: A Retrospective Immunohistochemical Analysis Using Tissue Microarray
}

\author{
Sung Han Kim, MD, PhD 1 \\ Weon Seo Park, MD, PhD² \\ Boram Park, MS ${ }^{3}$ \\ Jinsoo Chung, MD, PhD' \\ Jae Young Joung, $M D, P h D^{1}$ \\ Kang Hyun Lee, MD, PhD' \\ Ho Kyung Seo, MD, PhD'
}

\section{Purpose}

The purpose of this study was to identify prognostic tissue markers for several survival outcomes after radical nephroureterectomy among patients with upper urinary tract urothelial carcinoma using tissue microarray and immunohistochemistry.

\section{Materials and Methods}

Retrospectively, data of 162 non-metastatic patients with upper urinary tract urothelial carcinoma after radical nephroureterectomy between 2004 and 2016 were reviewed to determine intravesical recurrence-free survival (IVRFS), disease-free survival (DFS), and overall survival (OS). The expression of 27 tissue markers on a tissue microarray of radical nephroureterectomy samples and prognostic values of clinicopathological parameters were evaluated using immunohistochemistry and Cox proportional hazard models after adjusting for significant prognostic clinicopathological variables. The expression of all tissue markers was categorized into a binary group with continuous H-scores (0-300).

\section{Results}

Median follow-up was 53.4 months (range, 3.6 to 176.5 months); and, 58 (35.8\%), 48 (29.6\%), and 19 (11.7\%) bladder recurrence, disease progression, and all cause death, respectively, were identified. After adjusting for significant clinicopathological factors including intravesical instillation for bladder recurrence-free survival, pathologic $T$ category and intravesical instillation for disease progression-free survival , and pathologic T category for OS ( $p<0.05$ ), IVRFS was associated with epithelial cadherin (hazard ratio [HR], 0.49), epidermal growth factor receptor/erythroblastosis oncogene B (c-erb) (HR, 2.59), and retinoblastoma protein loss (HR, 1.85); DFS was associated with cyclin D1 (HR, 2.16) and highmolecular-weight cytokeratin (HR, 0.42); OS was associated with E-cadherin (HR, 0.34) and programmed cell death 1 ligand $(\mathrm{HR}, 13.42)(\mathrm{p}<0.05)$.

\section{Conclusion}

Several significant tissue markers were associated with survival outcomes in upper urinary tract urothelial carcinoma patients treated with radical nephroureterectomy.

\section{Key words}

Nephroureterectomy, Tissue marker, Microarray, Prognosis, Risk factor

\section{Introduction}

Upper urinary tract urothelial carcinoma (UTUC) accounts for approximately $5 \%-10 \%$ of all urothelial tumors [1]. The gold standard curative treatments for localized UTUC are radical nephroureterectomy (RNU) with ipsilateral bladder cuff removal for high-risk UTUC cases and either kidneysparing surgery with single segmental ureterectomy or endoscopic ablation for low-risk cases. Although the adequate surgical control of local tumor results in a long-term survival of $>80 \%$ to $90 \%$ [2], progression to regional nodal metastases and distant metastases results in estimated 5-year survival rates of $<30 \%$ and $<10 \%$, respectively [3]. These discrepant 
survival rates between localized and advanced states reflect the insufficient understanding of UTUC pathogenesis due to its rarity.

Studies on UTUC have been conducted for several decades to determine its similarities with urothelial carcinoma of the bladder. Various clinicopathological parameters related to prognoses have been found and used to establish multiple prognostic models to better predict the outcomes of UTUC after surgical therapy $[4,5]$. These factors include tumor stage, nuclear grade, positive lymph node status, and therapeutic modalities including intravesical instillation, as well as the heterogeneity and aggressiveness of the UTUC. The association of some factors with prognostic survivals has been confirmed, whereas the involvement of other factors, such as preoperative hydronephrosis and synchronous bladder tumor lesions, remained controversial due to the low tumor prevalence and the different surgical and chemotherapeutic protocols implemented by various institutions and clinicians [6-8].

Tissue microarray (TMA) of surgical specimens, and the expression of immunohistochemical tissue markers, may provide important clues to better understand the pathogenetic characteristics of UTUC and identify universal prognostic markers, which will aid in estimating more precise prognoses of UTUC after surgery. Therefore, in this study, 27 potential urothelial carcinoma-related tissue markers from previous urothelial carcinoma-related tissue studies and National Cancer Center bladder cancer panel [9-26] were applied immunohistochemically (IHC) to the TMA of specimens from patients with UTUC who underwent RNU, in order to identify any significant prognostic tissue markers of survival, including intravesical recurrence-free survival (IVRFS), disease-free survival (DFS), and overall survival (OS).

\section{Materials and Methods}

\section{Patient inclusion criteria and tissue samples}

A total of 162 patients with UTUC who underwent RNU including $46(28.4 \%)$ with a previous history of bladder cancer between 2002 and 2016 were selected, and their medical records and surgical specimens were retrospectively reviewed. Patients who had nonurothelial carcinoma histology, history of neoadjuvant chemotherapy, less than 3-month follow-up, and small tumor volume that was inadequate for the preparation of TMA were all excluded. All samples were transitional cell carcinoma specimens and reviewed blindly, retrospectively, and pathologically by an uropathologist
(W.S.P.) with 15 years of experience in accordance with the 2009 tumor, node, and metastasis (TNM) classification for UTUC and the 2004 World Health Organization (WHO)/ International Society of Urologic Pathologists (ISUP) consensus classification [10].

\section{IHC and assessment of TMA}

TMA and IHC assessments were performed in accordance with previously published study using TMAs [26]. TMA blocks were built using two representative tumor areas (2-mm core) and paired single normal control tissue from formalin-fixed, paraffin-embedded tumor material, and marked on standard hematoxylin and eosin-stained sections for the expressions of tissue markers [27].

The following 27 tissue biomarkers in the UTUC TMA were IHC stained: carbonic anhydrase (CA8 and CA9), platelet and endothelial cell adhesion molecule 1/CD31, epidermal growth factor receptor/erythroblastosis oncogene B (C-erb), cytokeratin (CK20 and CK5 \& 6), c-Myc, mitochondrially encoded cytochrome c oxidase II (COX2), epithelial cadherin 1 (E-cadherin), excision repair cross-complementation group 1 (ERCC1), hypoxia-inducible factor (HIF-1 $\alpha$ and HIF-2 $\alpha$ ), high-molecular-weight heparin (HMWCK) and low-molecular-weight heparin, programmed cell death 1 ligand (PD-L1), folate hydrolase 1/ prostate-specific membrane antigen 1 , phosphatase and tensin homolog, retinoblastoma protein (Rb), Ki-67, p53, cyclin D1 and D2, CD34 and 44, smooth muscle actin (SMA), and vascular endothelial growth factor $\mathrm{A}$. The staining condition was validated using negative and positive tissue controls for all antibodies (S1 and S2 Tables).

\section{Interpretation of IHC expression on TMA}

As described in the previously published study [26], the percentage of cells stained $(0 \%-100 \%)$ and the staining intensity were assessed within the nuclei and cytoplasm of malignant cells, and compared with those of the paired benign cells. The intensity of CD8 and SMA expression in the tumor environment was assessed. The cases were identified pathologically by a senior uropathologist (W.S.P.) blinded to the clinical outcome using the semi-quantitative $\mathrm{H}$-score (0-300), including the three-tier grading intensity score ( 0 for negative, $1+$ for weak, $2+$ for moderate, and $3+$ for strong). The intensity score was classified into two categories (negative [0-1] or positive [2-3]) to examine the concordance rate of the expressions of markers between different organs (Fig. 1) and different sites of the same organ (S1 Table). 


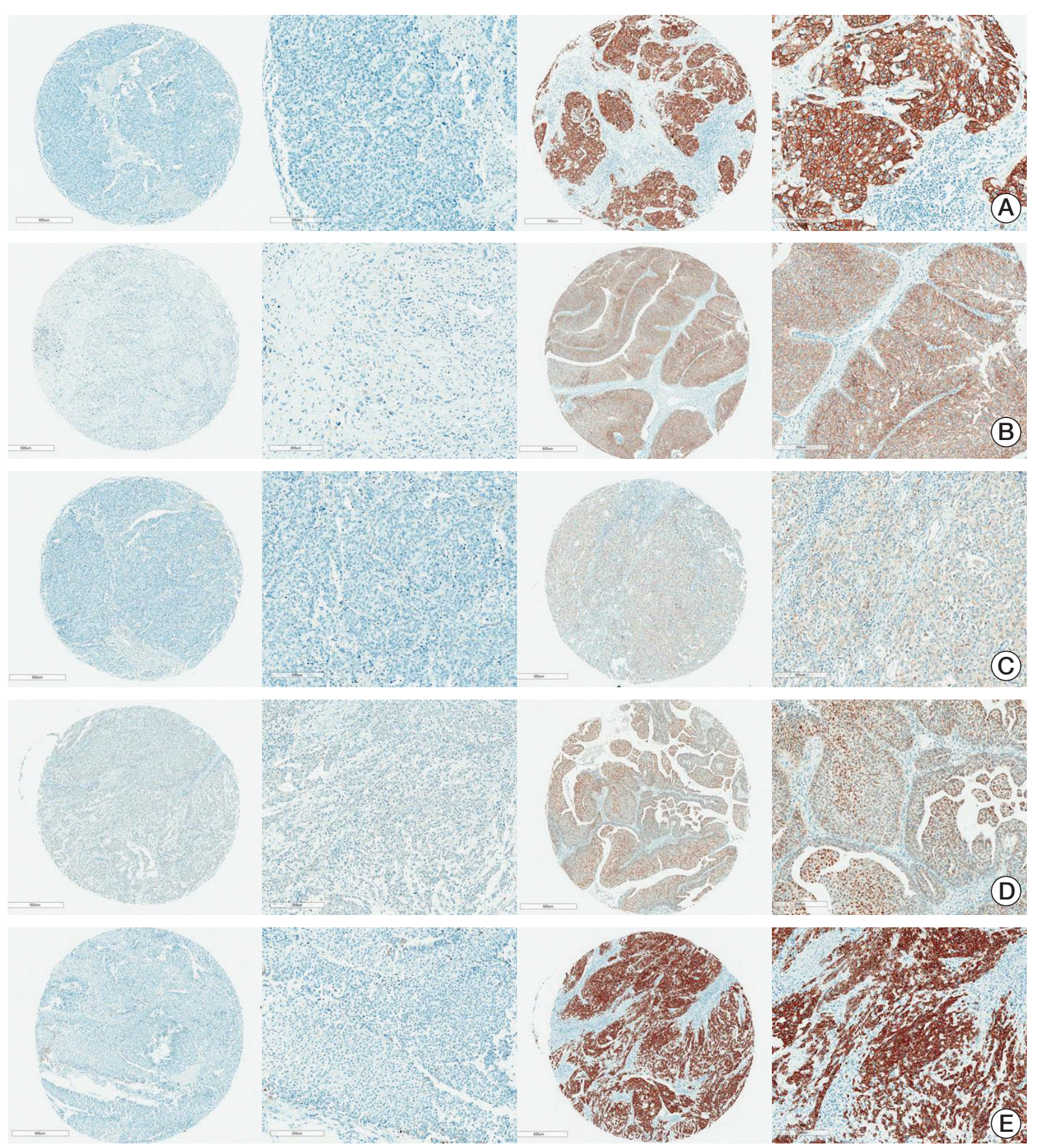

Fig. 1. Immunohistochemistry staining of five significant tissue markers according to their negative and positive expression: c-erb $(\times 35$ and $\times 100)(A)$, E-cadherin $(\times 35$ and $\times 100)(B)$, programmed cell death 1 ligand $(\times 35$ and $\times 100)(C)$, cyclin D1 $(\times 35$ and $\times 100)(\mathrm{D})$, and high-molecular-weight heparin $(\times 35$ and $\times 100)(\mathrm{E})$.

\section{Statistical analysis}

To examine the prognostic value of all 27 tissue biomarkers in terms of OS, DFS, and IVRFS, previously identified clinicopathological variables associated with prognosis were analyzed using a Cox proportional hazards model by a single medical statistician (B.P.). The IVRFS was defined as the free tumor status within the bladder and the DFS as the free disease status from the curative surgery until the detection of progression of the disease or recurrence of disease beyond 
Table 1. Baseline characteristics

\begin{tabular}{|c|c|}
\hline Characteristic & No. $(\%)(\mathrm{n}=162)$ \\
\hline Follow-up duration, median (range, mo) & $53.4(3.6-176.5)$ \\
\hline Age, mean $\pm S D(y r)$ & $66.5 \pm 10.4$ \\
\hline Body mass index, mean $\pm \mathrm{SD}\left(\mathrm{kg} / \mathrm{m}^{2}\right)$ & $24.0 \pm 3.4$ \\
\hline \multicolumn{2}{|l|}{ Sex } \\
\hline Male & $119(73.5)$ \\
\hline Female & $43(26.5)$ \\
\hline Hypertension & $74(45.7)$ \\
\hline Diabetes & $22(13.6)$ \\
\hline Other & $63(38.9)$ \\
\hline \multicolumn{2}{|l|}{ ASA score } \\
\hline 1 & $34(22.4)$ \\
\hline 2 & $105(69.1)$ \\
\hline 3 & $13(8.5)$ \\
\hline \multicolumn{2}{|l|}{ Nephroureterectomy method } \\
\hline Open & $90(55.6)$ \\
\hline Laparoscopy & $72(44.4)$ \\
\hline \multicolumn{2}{|l|}{ Previous history } \\
\hline No & $111(68.5)$ \\
\hline Previous bladder tumor history & $46(28.4)$ \\
\hline Concomitant bladder tumor history & $5(3.1)$ \\
\hline Intravesical instillation & $59(36.4)$ \\
\hline \multicolumn{2}{|l|}{ Tumor location } \\
\hline Renal pelvis & $65(40.4)$ \\
\hline Proximal & $7(4.4)$ \\
\hline Mid & $18(11.2)$ \\
\hline Distal & $38(23.6)$ \\
\hline More than two sites & $33(20.5)$ \\
\hline \multicolumn{2}{|l|}{ Tumor grade } \\
\hline I & $5(3.3)$ \\
\hline II & $55(36.2)$ \\
\hline III & $92(60.5)$ \\
\hline \multicolumn{2}{|l|}{ ISUP } \\
\hline Low & $32(21.1)$ \\
\hline High & $120(78.9)$ \\
\hline \multicolumn{2}{|l|}{ Pathologic $\mathrm{T}$ category } \\
\hline $\mathrm{Ta}$ & $8(5.0)$ \\
\hline $\mathrm{T} 1$ & $25(15.7)$ \\
\hline $\mathrm{T} 2$ & $40(25.2)$ \\
\hline T3 & $76(47.8)$ \\
\hline $\mathrm{T} 4$ & $8(5.0)$ \\
\hline CIS only & $2(1.3)$ \\
\hline \multicolumn{2}{|l|}{ Pathologic N category } \\
\hline $\mathrm{Nx}$ & $96(59.3)$ \\
\hline N0 & $53(32.7)$ \\
\hline N1 & $10(6.2)$ \\
\hline N2 & $3(1.9)$ \\
\hline \multicolumn{2}{|l|}{ Tumor size $(\mathrm{cm})$} \\
\hline $0-1.5$ & $13(8.1)$ \\
\hline $1.5-2.5$ & $27(16.9)$ \\
\hline$>2.5$ & $120(75.0)$ \\
\hline
\end{tabular}

(Continued)
Table 1. Continued

\begin{tabular}{lc} 
Characteristic & No. (\%) (n=162) \\
\hline Adjuvant chemotherapy & $72(44.4)$ \\
Bladder recurrence & $58(35.8)$ \\
Disease progression & $48(29.6)$ \\
Cause-specific death & \\
$\quad$ Upper tract & $4(21.1)$ \\
Bladder & $2(10.5)$ \\
Others & $13(68.4)$ \\
\hline
\end{tabular}

SD, standard deviation; ASA, American Society of Anesthesiologists; ISUP, International Society of Urologic Pathologists; CIS, carcinoma in situ.

the intravesical recurrence. The continuous tissue biomarkers were categorized into a binary group (low, high) using a cutoff value determined using the Contal and O'Quigley method [28]. After examining the significance of clinicopathological variables in the univariate model (S3 Table), four variables (age, intravesical instillation, ISUP, and pathologic [pT] category) were included in a multivariable clinicopathological model, and the final adjusted parameters were determined using a backward variable selection method with an elimination criterion of 0.10 . After adjusting for significant clinicopathological variables, the 27 tissue biomarkers were respectively evaluated in the final multivariable model for IVRFS, DFS, and OS (S3 and S4 Tables). The results were presented as hazard ratios (HR) with their 95\% confidence intervals (CI). The survival curves were plotted using survival probability estimated from the final multivariable model. p-values of less than 0.05 were considered significant. All statistical analyses were performed using SAS ver. 9.4 (SAS Institute Inc., Cary, NC).

\section{Ethical statement}

This retrospective study was approved by the Institutional Review Board of the National Cancer Center (IRB No. NCC 2018-0166), and the requirement for informed consent was waived owing to the retrospective nature of the study. All patient data were anonymized and de-identified prior to our analysis. All study protocols were performed in accordance with the tenets of the Declaration of Helsinki. 
Table 2. Univariable and multivariable Cox proportional hazard model of high or low expression level for IVRFS, DFS, and OS after adjusted by significant prognostic clinicopathologic variables for each survival

\begin{tabular}{|c|c|c|c|c|c|c|c|}
\hline & \multirow{2}{*}{$\begin{array}{l}\text { Cut- } \\
\text { point }\end{array}$} & \multirow{2}{*}{$\begin{array}{c}\text { No. } \\
(n=162)\end{array}$} & \multirow{2}{*}{ Event $(\%)$} & \multicolumn{2}{|c|}{ Univariable } & \multicolumn{2}{|c|}{ Multivariable } \\
\hline & & & & HR $(95 \%$ CI $)$ & p-value & $\mathrm{HR}(95 \% \mathrm{CI})$ & p-value \\
\hline \multicolumn{8}{|c|}{$\operatorname{IVRFS}^{\text {a) }}(n=162$, event=58) } \\
\hline \multicolumn{8}{|c|}{ C-erb } \\
\hline Low & 0 & 141 & $52(36.9)$ & 1 (reference) & \multicolumn{3}{|c|}{1 (reference) } \\
\hline High & $>0$ & 21 & $6(28.6)$ & $0.74(0.32-1.72)$ & 0.483 & $2.59(1.05-6.39)$ & 0.039 \\
\hline \multicolumn{8}{|l|}{$\mathrm{C}$-myc } \\
\hline Low & $\leq 30$ & 82 & $35(42.7)$ & 1 (reference) & \multicolumn{3}{|c|}{1 (reference) } \\
\hline High & $>30$ & 80 & $23(28.8)$ & $0.56(0.33-0.95)$ & 0.031 & $0.88(0.52-1.51)$ & 0.650 \\
\hline \multicolumn{8}{|c|}{ E-cadherin } \\
\hline Low & $\leq 270$ & 119 & $45(37.8)$ & 1 (reference) & \multicolumn{3}{|c|}{1 (reference) } \\
\hline High & $>270$ & 43 & $13(30.2)$ & $0.69(0.37-1.27)$ & 0.231 & $0.49(0.26-0.91)$ & 0.025 \\
\hline \multicolumn{8}{|l|}{ ERCC1 } \\
\hline Low & $\leq 130$ & 63 & $31(49.2)$ & 1 (reference) & \multicolumn{3}{|c|}{1 (reference) } \\
\hline High & $>130$ & 99 & $27(27.3)$ & $0.5(0.3-0.83)$ & 0.008 & $1.06(0.63-1.8)$ & 0.818 \\
\hline \multicolumn{8}{|l|}{$\mathrm{Rb}$ loss } \\
\hline Low & $\leq 20$ & 66 & $22(33.3)$ & 1 (reference) & \multicolumn{3}{|c|}{1 (reference) } \\
\hline High & $>20$ & 96 & $36(37.5)$ & $1.21(0.71-2.06)$ & 0.478 & $1.85(1.07-3.19)$ & 0.028 \\
\hline \multicolumn{8}{|l|}{ VEGF } \\
\hline Low & $\leq 170$ & 75 & $35(46.7)$ & 1 (reference) & \multicolumn{3}{|c|}{1 (reference) } \\
\hline High & $>170$ & 87 & $23(26.4)$ & $0.45(0.27-0.77)$ & 0.003 & $0.66(0.39-1.13)$ & 0.132 \\
\hline \multicolumn{8}{|c|}{$\operatorname{DFS}^{b)}(n=162$, event $=48)$} \\
\hline \multicolumn{8}{|c|}{$\mathrm{COX} 2$} \\
\hline Low & $<300$ & 110 & $27(24.6)$ & 1 (reference) & \multicolumn{3}{|c|}{1 (reference) } \\
\hline High & $\geq 300$ & 52 & $21(40.4)$ & $1.9(1.08-3.37)$ & 0.027 & $1.55(0.87-2.75)$ & 0.137 \\
\hline \multicolumn{8}{|c|}{ Cyclin D1 } \\
\hline Low & $\leq 40$ & 81 & $17(21.0)$ & 1 (reference) & \multicolumn{3}{|c|}{1 (reference) } \\
\hline High & $>40$ & 81 & $31(38.3)$ & $2.07(1.15-3.75)$ & 0.016 & $2.16(1.19-3.94)$ & 0.011 \\
\hline \multicolumn{8}{|c|}{ HMWCK } \\
\hline Low & $\leq 130$ & 88 & $34(38.6)$ & 1 (reference) & \multicolumn{3}{|c|}{1 (reference) } \\
\hline High & $>130$ & 74 & $14(18.9)$ & $0.39(0.21-0.72)$ & 0.003 & $0.42(0.22-0.79)$ & 0.007 \\
\hline PD-L1 & & & & & & & \\
\hline Low & 0 & 157 & $45(28.7)$ & 1 (reference) & & 1 (reference) & \\
\hline High & $>0$ & 5 & $3(60.0)$ & $4.05(1.25-13.16)$ & 0.020 & $2.26(0.69-7.37)$ & 0.178 \\
\hline $\mathrm{OS}^{\mathrm{c})}(\mathrm{n}=16$ & & & & & & & \\
\hline E-cadhe & & & & & & & \\
\hline Low & $<150$ & 85 & $14(16.5)$ & 1 (reference) & & 1 (reference) & \\
\hline High & $>150$ & 77 & $5(6.5)$ & $0.37(0.13-1.02)$ & 0.055 & $0.34(0.12-0.94)$ & 0.038 \\
\hline PD-L1 & & & & & & & \\
\hline Low & 0 & 157 & $16(10.2)$ & 1 (reference) & & 1 (reference) & \\
\hline High & $>0$ & 5 & $3(60.0)$ & $17.2(4.68-63.18)$ & $<0.001$ & $13.42(3.64-49.43)$ & $<0.001$ \\
\hline
\end{tabular}

IVRFS, intravescial recurrence-free survival; DFS, disease-free survival; OS, overall survival; $\mathrm{HR}$, hazard ratio; $\mathrm{CI}$, confidence interval; ERCC1, ERCC excision repair 1; Rb, retinoblastoma; VEGF, vascular endothelial growth factor; COX2, cytochrome coxidase II; HMWCK, high-molecular-weight heparin; PD-L1, programmed cell death 1 ligand. a)Adjusted for intravesical instillation in multivariable bladder recurrence-free survival model, b)Adjusted for intravesical instillation and pTstage in multivariable progression-free survival model, c)Adjusted for pTstage in multivariable OS model. 


\section{Results}

The mean \pm standard deviation age of the patients was $66.5 \pm$ 10.4 years (men, 119, 73.5\%). The rate of pathologic Tis, Ta, and $\mathrm{T} 1$ was $22.0 \%(\mathrm{n}=35)$, while those of $\mathrm{pN1}$-pT2 cateogry and higher ISUP nuclear grade were $8.1 \%(\mathrm{n}=13)$ and $78.9 \%$ $(\mathrm{n}=120)$, respectively. There were 72 patients $(44.4 \%)$ who received adjuvant chemotherapy after surgery. Within a median follow-up of 53.4 (range, 3.6 to 176.5 months), patients with intravesical recurrence (58 cases, $35.8 \%$ ), disease recurrence beyond the bladder (48 cases, $29.6 \%$ ), and all cause death (19 cases, 11.7\%) including six cancer-specific deaths $(31.6 \%)$ were identified. The remaining clinicopathological characteristics of the patients and the results of IHC staining for each tissue biomarker are summarized in Table 1 and S1 Table, respectively.

According to the univariable analysis, the clinicopathological parameters associated with survival prognosis in terms of IVRFS were intravesical instillation after surgery (HR, 22.08; $95 \%$ CI, 9.96 to 48.94 ), ISUP nuclear grade (low vs. high; HR, 0.57; 95\% CI, 0.33 to 0.98), and pathologic T category (pTa, pT1, and pTis vs. pT2-pT4; HR, $0.49 ; 95 \%$ CI, 0.28 to 0.84 ). With regard to DFS, intravesical instillation (HR, $0.22 ; 95 \% \mathrm{CI}, 0.10$ to 0.49 ), ISUP nuclear grade (low vs. high; $\mathrm{HR}, 3.02 ; 95 \% \mathrm{CI}, 1.19$ to 7.68$)$, and pathologic T category (pTa, pT1, and pTis vs. pT2-pT4; HR, 8.72; 95\% CI, 2.11 to 35.95) were significant risk factors. As regards OS, pathologic $\mathrm{T}$ category (pTa, pT1, and pTis vs. pT2-pT4; HR, 6.83; 95\% CI, 0.91 to 51.35) only showed significant tendency (S3 Table). After four significant prognostic variables (age, intravesical instillation, ISUP, and pT category) were included in the multivariable model with tissue markers, only intravesical instillation in the IVRFS model, intravesical instillation (HR, $0.27 ; 95 \% \mathrm{CI}, 0.12$ to $0.59 ; \mathrm{p}=0.001)$ and $\mathrm{pT}$ category (HR, 6.54; 95\% CI, 1.58 to 27.16; $\mathrm{p}=0.010$ ) in the DFS model, and pT stage in the OS model remained significant (S3 Table).

With regard to the significant tissue markers for each survival model (Table 2), IVBRFS was associated with E-cadherin (HR, 0.49; 95\% CI, 0.26 to 0.91 ), c-erb (HR, 2.59; 95\% CI, 1.05 to 6.39 ), and $\mathrm{Rb}$ loss (HR, 1.85; 95\% CI, 1.07 to 3.19 ) (Table 2); DFS was associated with cyclin D1 (HR, 2.16; 95\% CI, 1.19 to 3.94 ) and HMWCK (HR, $0.42 ; 95 \%$ CI, 0.22 to 0.79 ) (Table 2); and OS was associated with E-cadherin (HR, 0.34; 95\% CI, 0.12 to 0.94 ) and PD-L1 (HR, 13.42; 95\% CI, 3.64 to 49.43) in multivariable models including the tissue marker intensity scores (either low or high) of the enrolled patients $(p<0.05)$ (Table 2). The Kaplan-Meier survival curves also showed significant differences between the two groups with different expression levels for c-erb, E-cadherin, and $\mathrm{Rb}$ loss for IVRFS (Fig. 2A); cyclin D1 and HMWCK for DFS (Fig. 2B); and E-cadherin and PD-L1 for OS (Fig. 2C) $(\mathrm{p}<0.05)$.
An additional subgroup analysis was conducted among 111 patients with no prior or concurrent bladder cancer history, to determine the significant prognostic tissue markers associated with IVRFS, DFS, and OS. Results showed that there was a significant difference in the tissue analysis results of all patients (Table 3). The multivariable analyses of the tumor marker expression levels (either high or low) showed that none of these markers had significant predictive power in IVRFS after adjusting for intravesical instillation. Whereas only cyclin D1 (HR, 2.1; 95\% CI, 1.07 to 4.10) and HMWCK (HR, 0.48; 95\% CI, 0.24 to 0.99) in DFS, after adjusting for intravesical instillation, and PD-L1 (HR, 11.27; 95\% CI, 2.95 to 43.02 ) and E-cadherin (HR, $0.35 ; 95 \% \mathrm{CI}, 0.12$ to 0.99 ) in OS, after adjusting for pathologic $\mathrm{T}$ category remained significant factors $(\mathrm{p}<0.05)$.

\section{Discussion}

The therapeutic, prognostic, and postoperative follow-up modalities including neo/ adjuvant protocols of UTUC were similar to those of bladder-originated urothelial carcinoma. However, the natural history and etiology of UTUC differed from those of bladder cancer with more invasive diagnostic protocols and poorer survival outcomes [1]. More prognostic factors including postoperative pathologic stage, grade, lymph node invasion, lymphovascular invasion, and tumor location have been identified for UTUC than for bladder cancer. Meanwhile, the presence of previous bladder cancer history distinguished UTUC from bladder cancer $[4,5,10,14,17,29]$.

Recent studies with TMA of RNU specimens have attempted to define more stratified and precise classification methods based on tissue and genetic markers similar to other cancers $[10,21]$. Immunohistochemistry using TMA was widely used to better predict the therapeutic outcome of surgical and systemic medical interventions [9]. More accurate subtyping of multi-heterogeneous and mutated cancers has resulted from the understanding of biopsied and surgical tissues obtained from patients with cancer and the expression of each tissue marker in these specimens. This leads to the possibility of individualizing treatment options according to the molecular profile of the tumor, thus increasing prognostic survival and curative rate.

This study investigated the significant tissue markers of three prognostic outcomes (intravesical recurrence, disease recurrence beyond the bladder, and death) with adjustment for the significant prognostic clinicopathological parameters among the 162 patients with and without previous bladder cancer history and several potential prognostic tissue markers among the selected 27 known tissue markers relating to 


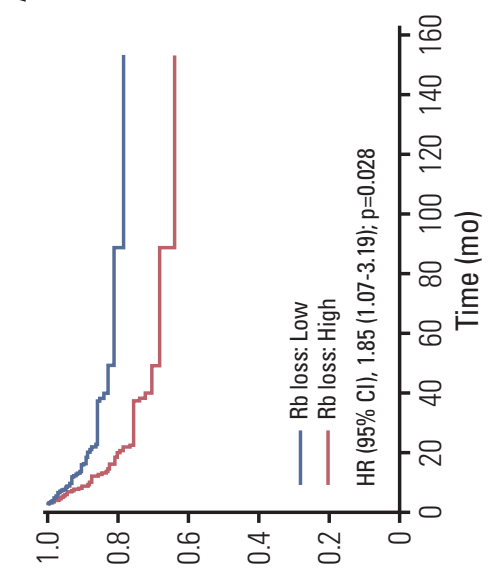

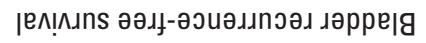

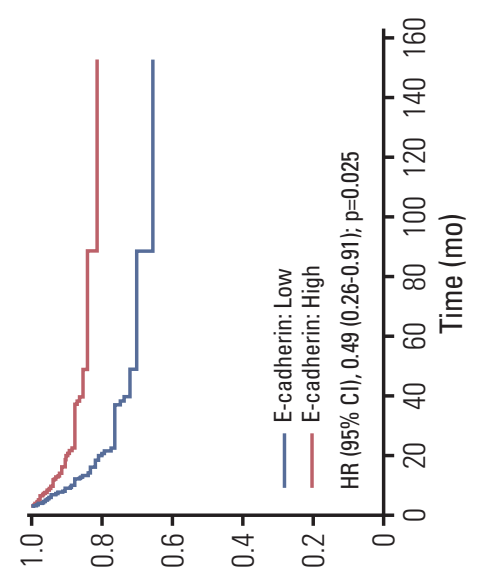

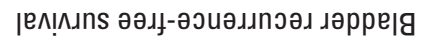

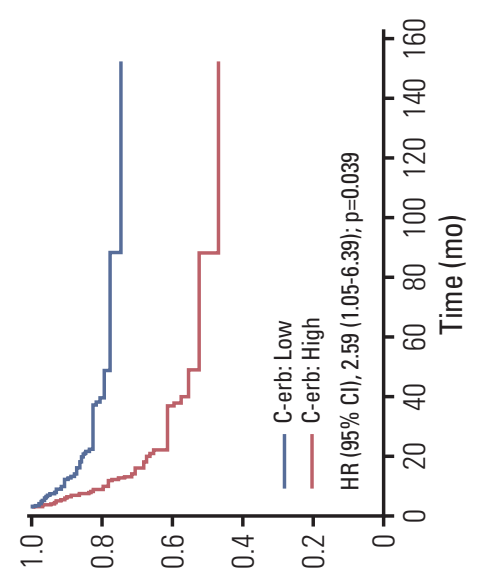<smiles>Ic1ccccc1</smiles>

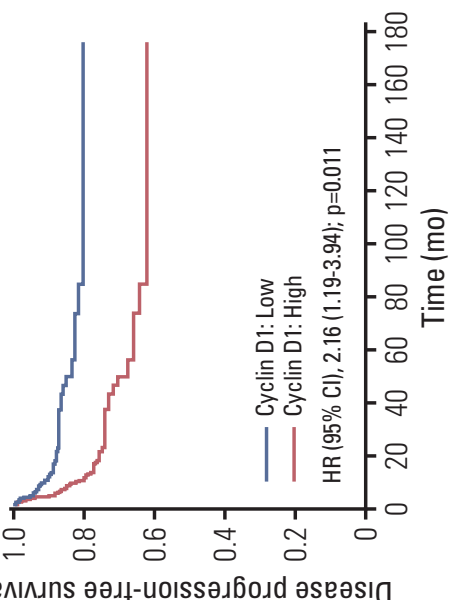

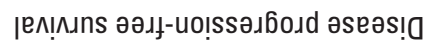

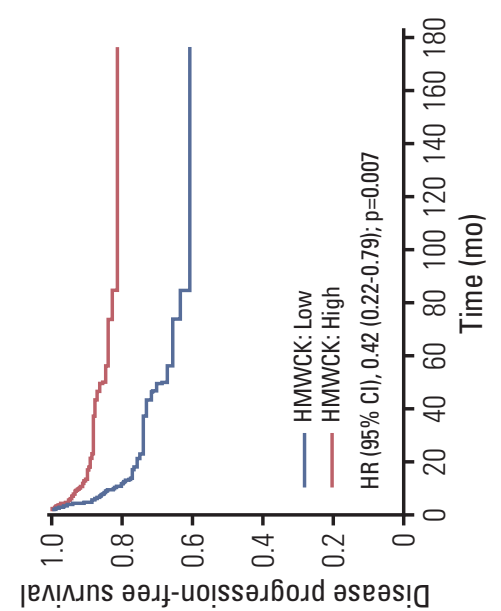

(
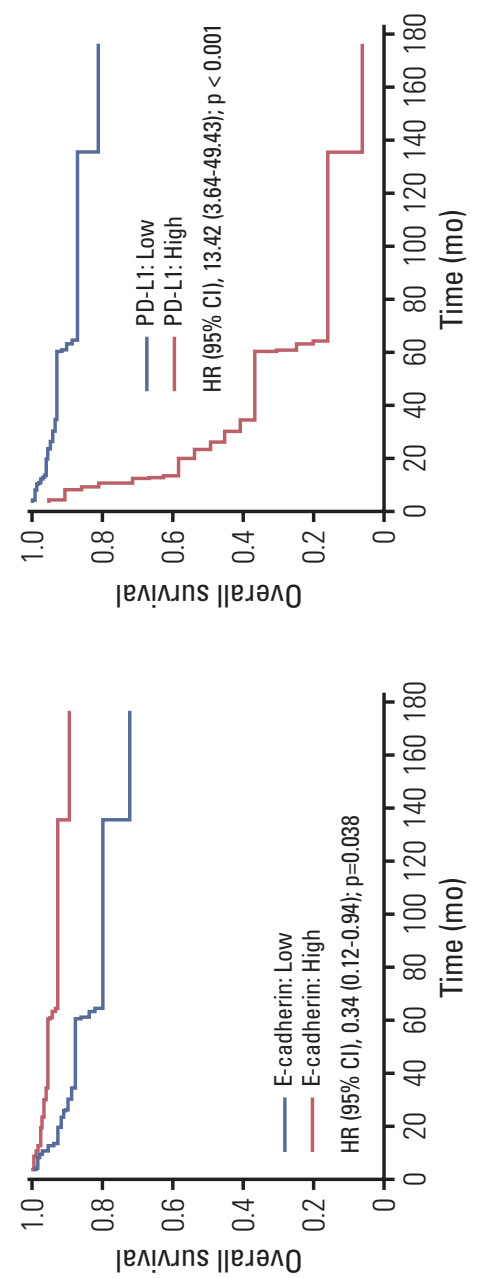
Table 3. Univariable and multivariable Cox proportional hazard model of high or low expression level for BRFS, DPFS, and OS in only patients with no prior or concurrent bladder cancer history

\begin{tabular}{|c|c|c|c|c|c|c|c|}
\hline & \multirow{2}{*}{$\begin{array}{l}\text { Cut- } \\
\text { point }\end{array}$} & \multirow{2}{*}{$\begin{array}{c}\text { No. } \\
(n=111)\end{array}$} & \multirow{2}{*}{ Event $(\%)$} & \multicolumn{2}{|c|}{ Univariable } & \multicolumn{2}{|c|}{ Multivariable } \\
\hline & & & & HR $(95 \%$ CI $)$ & p-value & HR $(95 \% \mathrm{CI})$ & p-value \\
\hline \multicolumn{8}{|c|}{ BRFS $^{\text {a) }}(n=111$, event=36) } \\
\hline \multicolumn{8}{|l|}{ C-erb } \\
\hline Low & 0 & 95 & $30(31.6)$ & 1 (reference) & \multicolumn{3}{|c|}{1 (reference) } \\
\hline High & $>0$ & 16 & $6(37.5)$ & $1.24(0.52-2.99)$ & 0.629 & $2.55(1.01-6.41)$ & 0.047 \\
\hline \multicolumn{8}{|l|}{$\mathrm{C}$-myc } \\
\hline Low & $\leq 30$ & 58 & $23(39.7)$ & 1 (reference) & \multicolumn{3}{|c|}{1 (reference) } \\
\hline High & $>30$ & 53 & $13(24.5)$ & $0.54(0.27-1.06)$ & 0.072 & $1.02(0.50-2.05)$ & 0.964 \\
\hline \multicolumn{8}{|c|}{ E-cadherin } \\
\hline Low & $\leq 270$ & 84 & $27(32.1)$ & 1 (reference) & \multicolumn{3}{|c|}{1 (reference) } \\
\hline High & $>270$ & 27 & $9(33.3)$ & $1.07(0.50-2.27)$ & 0.867 & $1.28(0.60-2.76)$ & 0.522 \\
\hline \multicolumn{8}{|l|}{ ERCC1 } \\
\hline Low & $\leq 130$ & 42 & $17(40.5)$ & 1 (reference) & \multicolumn{3}{|c|}{1 (reference) } \\
\hline High & $>130$ & 69 & $19(27.5)$ & $0.61(0.32-1.17)$ & 0.138 & $1.23(0.62-2.42)$ & 0.554 \\
\hline \multicolumn{8}{|l|}{$\mathrm{Rb}$} \\
\hline Low & $\leq 20$ & 43 & $12(27.9)$ & 1 (reference) & \multicolumn{3}{|c|}{1 (reference) } \\
\hline High & $>20$ & 68 & $24(35.3)$ & $1.37(0.68-2.75)$ & 0.375 & $1.74(0.85-3.54)$ & 0.129 \\
\hline \multicolumn{8}{|l|}{ VEGF } \\
\hline Low & $\leq 170$ & 47 & $19(40.4)$ & 1 (reference) & \multicolumn{3}{|c|}{1 (reference) } \\
\hline High & $>170$ & 64 & $17(26.6)$ & $0.57(0.30-1.11)$ & 0.097 & $0.97(0.50-1.89)$ & 0.925 \\
\hline \multicolumn{8}{|c|}{ DPFS $^{b)}(n=111$, event $=34)$} \\
\hline \multicolumn{8}{|l|}{ COX2 } \\
\hline Low & $<300$ & 71 & $18(25.4)$ & 1 (reference) & \multicolumn{3}{|c|}{1 (reference) } \\
\hline High & 300 & 40 & $16(40.0)$ & $1.85(0.94-3.63)$ & 0.074 & $1.53(0.77-3.01)$ & 0.223 \\
\hline \multicolumn{8}{|c|}{ Cyclin D1 } \\
\hline Low & $\leq 40$ & 53 & $12(22.6)$ & 1 (reference) & \multicolumn{3}{|c|}{1 (reference) } \\
\hline High & $>40$ & 58 & $22(37.9)$ & $2.04(1.01-4.14)$ & 0.048 & $2.33(1.14-4.75)$ & 0.020 \\
\hline \multicolumn{8}{|c|}{ HMWCK } \\
\hline Low & $\leq 130$ & 59 & $22(37.3)$ & 1 (reference) & \multicolumn{3}{|c|}{1 (reference) } \\
\hline High & $>130$ & 52 & $12(23.1)$ & $0.50(0.25-1.02)$ & 0.057 & $0.48(0.24-0.99)$ & 0.047 \\
\hline PD-L1 & & & & & & & \\
\hline Low & 0 & 106 & $31(29.3)$ & 1 (reference) & & 1 (reference) & \\
\hline High & $>0$ & 5 & $3(60.0)$ & $4.05(1.22-13.48)$ & 0.023 & $2.32(0.69-7.74)$ & 0.173 \\
\hline $\mathrm{OS}^{\mathrm{c})}(\mathrm{n}=11$ & & & & & & & \\
\hline E-cadhe & & & & & & & \\
\hline Low & $\leq 150$ & 57 & $12(21.1)$ & 1 (reference) & & 1 (reference) & \\
\hline High & $>150$ & 54 & $5(9.3)$ & $0.41(0.14-1.15)$ & 0.090 & $0.35(0.12-0.99)$ & 0.048 \\
\hline PD-L1 & & & & & & & \\
\hline Low & 0 & 106 & $14(13.2)$ & 1 (reference) & & 1 (reference) & \\
\hline High & $>0$ & 5 & $3(60.0)$ & $13.82(3.64-52.49)$ & $<0.001$ & $10.8(2.83-41.25)$ & 0.001 \\
\hline
\end{tabular}

BRFS, bladder recurrence-free survival; DPFS, disease progression-free survival; OS, overall survival; $\mathrm{HR}$, hazard ratio; $\mathrm{CI}$, confidence interval; C-erb, epidermal growth factor receptor; ERCC1, ERCC excision repair 1; Rb, retinoblastoma; VEGF, vascular endothelial growth factor; COX2, cytochrome c oxidase II; HMWCK, high-molecular-weight heparin; PD-L1, programmed cell death 1 ligand. ${ }^{\text {a) }}$ Adjusted for intravesical instillation in multivariable BRFS model, b)Adjusted for age, intravesical instillation and pTstage in multivariable progression-free survival model, ${ }^{c}$ Adjusted for pTstage in multivariable OS model. 
UTUC, bladder urothelial carcinoma, and known therapeutic targets such as checkpoint inhibitors, even after adjustment for the significant prognostic clinicopathological parameters $(p<0.05)$ (Tables 2 and 3, Fig. 2). In the sub-analyses, about 46 patients with a previous history of bladder cancer also showed some useful prognostic markers of survivals even after adjusting for TMA $(\mathrm{p}<0.05)$ (Table 3$)$.

Previous studies showed that cyclin D1 was a significant predictor for DFS in patients with bladder urothelial carcinoma and UTUC $[10,11]$. Moreover, it played an important role in the early onset of urothelial tumorigenesis, driving cell proliferation and infiltration [10], and was correlated with prognostic clinicopathological parameters, such as TNM stage and nuclear grade, in bladder urothelial carcinoma [11]. Additionally, cyclin D1 was significantly associated with tumor recurrence, thus decreasing DFS [12]. By contrast, this study also showed that cyclin D1 was associated with DFS (HR, 2.16; $\mathrm{p}=0.011$ ) (Table 2).

PD-L1 marker is target receptor in the tumor cell membrane and tumor-infiltrating mononuclear cells to anti-PDL1 checkpoint inhibitor, and it was shown to be a significantly strong marker for OS (HR, 13.42) in this study (Table 2), similar to the report of other published studies [12-14]. The PD-1/PD-L1 pathway has been highlighted since the last decade; that tumor cells can evade the host immune surveillance through the overexpression of the checkpoint ligands on tumor cells such as PD-L1 [13,14]. This immune suppressing operation of cancer cells involve immune cell intrinsic checkpoint ligands that are induced on the surface of activated $\mathrm{T}$ cells resulting in T-cell dysfunction, which impairs anti-tumor immunity. Many immune agents have been developed in clinical use for many malignancies, resulting in improved prognostic survivals.

The current immune checkpoint inhibitor targeting the PDL1 expression has proven its potential therapeutic effect with longer survival in patients with metastatic urothelial carcinoma. The expression of PD-L1 was usually tested as a predictive marker to anticipate the response of checkpoint inhibitor. In addition, this study showed that the expression of PD-L1 in tumor cells was significantly related to the OS for poor prognostic marker (HR, 13.42; 95\% CI, 3.64 to 49.43) after adjusting the significant prognostic clinicopathological parameters in localized UTUC $(\mathrm{p}<0.01)$ (Table 2). The Kaplan-Meier curve of OS also supported the significantly poorer prognostic outcomes in positive PD-L1 group $(n=5)$ than in the negative PD-L1 group ( $\mathrm{n}=175$ ) (Fig. 2C). Although the number of deaths in the positive group looked small compared to the 16 deaths in the negative PD-L1 group, the proportional survival rate of PD-L1 positive was higher $(60 \%)$ than the negative PD-L1 negative group (10.2\%). This implies that the PD-L1 inhibitor might be an efficacious therapy, even in patients with localized UTUC who may be unsuitable candidates for surgery due to major comorbidities. The positive expression level of PD-L1 was observed in only $3.1 \%$ of the study population $(n=5)$. The method to evaluate PD-L1 expression in urothelial cancer has not been standardized. Several antibodies have been used for different targets (e.g., tumor cell or immune cell or combine) with different targets. The current checkpoint inhibitor was approved for use only in patients with locally advanced or metastatic urothelial carcinoma as a second-line therapy after failure of first-line chemotherapy in Korea, and the cut-off point of expression level was 5\% [14]. This is the first study to report the expression levels of PD-L1 in patients with UTUC after RNU.

The E-cadherin marker was considered as a favorable marker in both IVRFS and OS ( $p<0.05)$, in spite of its loss of marginal significance when presented as a continuous value of expression ( $\mathrm{p}=0.051$ ) (Table 2). E-cadherin is an important prognostic factor for UTUC; loss of E-cadherin expression or weak E-cadherin expression is related to IVRFS and DFS [15]. A recent IHC study by Cho et al. [16] using TMA of RNU showed the correlation between loss of E-cadherin levels and epithelial-mesenchymal transition (EMT) phenotypes such as wild type, incomplete type (loss of E-cadherin and negative for vimentin), and complete type (loss of E-cadherin and positive for vimentin). The EMT complete type was an independent prognostic factor for extra-bladder recurrence-free survival and OS, which was similar to the results of DFS and OS in this study conducted among 162 patients.

$\mathrm{Rb}$ loss [17] and c-erb [18] were well-known prognostic markers in upper urinary and bladder urothelial carcinoma. The loss of RB expression is a well-known invasive pathogenetic marker in bladder urothelial carcinoma, resulting in progression to muscle invasive bladder cancer from noninvasive bladder cancer, often resulting in resistance to cisplatin chemotherapy $[19,20]$. However, no previous study has identified the prognostic role of the $\mathrm{Rb}$ tissue marker in UTUC until this study identified its unfavourable role in IVRFS (HR, 1.85) (Table 2). C-erbB2, another significant factor identified for the first time in UTUC, has been reported in a literature on bladder urothelial carcinoma for its prognostic role in IVRFS and its relation to histologic grade. This study supported this finding, in which the tumor grade and pathologic $\mathrm{N}$ stage were significantly related to the c-erbB2, marker $(\mathrm{p}<0.05)$ (S3 Table) [21,22]. A Spanish study [23] using 61 TMAs of RNU showed that c-erbB2 was negatively associated with cancer-specific death and disease progression. This finding also supports our results, indicating that c-erbB2, was the only significant unfavourable risk factor (HR, 2.59) for IVRFS (Table 2).

The last significant HMWCK marker for DFS is a routine tissue marker with Ki-67 in bladder urothelial carcinoma, with frequent expression $(>60 \%)$ in all invasive tumors 
$[25,26]$. It is an urothelial carcinoma-specific marker and frequently used to distinguish between urothelial carcinoma and differentiated prostatic carcinoma that mimics urothelial carcinoma [10].

This study had some limitations besides the retrospective design which included only few patients with UTUC and the arbitrary binary cut-off points of expression levels. Some other important prognostic clinicopathological parameters such as intraoperative complication was not considered in this study due to the absence of the Clavien grade 3 or 4 complication, whereas the large-numbered Connolly and Rochester study demonstrated the safety of nephroureterectomy procedure with a report of only $4 \%$ of Clavien grade 3 or 4 complications [29]. These limitations might interfere with the significance of this study. More important markers associated with basal and luminal urothelial carcinoma of the bladder, which were not examined in this study, should be considered in future trials using larger cohorts of UTUC. However, this 27 IHC analytic report will help determine the molecular diagnostic and predictive biomarkers of rare UTUC, similar to those of bladder urothelial carcinoma. These might be used as baseline data to further validate the therapeutic roles of currently used targeted agents such as fibroblast growth factor receptor 3, human epidermal growth factor receptor 2, and PD-1 / PD-L1 in bladder urothelial carcinoma.

In conclusion, this study identified several significant prognostic tissue markers in UTUC TMAs, which would help better understand the pathogenesis and prognostic outcomes of UTUC. Some of the tissue markers identified in this study were the first reported prognostic factors for UTUC after RNU.

\section{Electronic Supplementary Material}

Supplementary materials are available at Cancer Research and Treatment website (https:// www.e-crt.org).

\section{Conflicts of Interest}

Conflict of interest relevant to this article was not reported.

\section{Acknowledgments}

We thank Ms. Seung Hee Ham for creating the database and performing the sorting processes. This study was supported by the Korean National Cancer Center Grants (Nos. 1613231 and 1810242-1).

\section{References}

1. Ploeg M, Aben KK, Kiemeney LA. The present and future burden of urinary bladder cancer in the world. World J Urol. 2009;27:289-93.

2. Roupret M, Babjuk M, Comperat E, Zigeuner R, Sylvester RJ, Burger M, et al. European Association of Urology guidelines on upper urinary tract urothelial cell carcinoma: 2015 update. Eur Urol. 2015;68:868-79.

3. Raman JD, Scherr DS. Management of patients with upper urinary tract transitional cell carcinoma. Nat Clin Pract Urol. 2007;4:432-43.

4. Cha EK, Shariat SF, Kormaksson M, Novara G, Chromecki TF, Scherr DS, et al. Predicting clinical outcomes after radical nephroureterectomy for upper tract urothelial carcinoma. Eur Urol. 2012;61:818-25.

5. Yates DR, Hupertan V, Colin P, Ouzzane A, Descazeaud A, Long JA, et al. Cancer-specific survival after radical nephroureterectomy for upper urinary tract urothelial carcinoma: proposal and multi-institutional validation of a post-operative nomogram. Br J Cancer. 2012;106:1083-8.

6. Chung PH, Krabbe LM, Darwish OM, Westerman ME, Bagrodia A, Gayed BA, et al. Degree of hydronephrosis predicts adverse pathological features and worse oncologic outcomes in patients with high-grade urothelial carcinoma of the upper urinary tract. Urol Oncol. 2014;32:981-8.

7. Hwang I, Jung SI, Nam DH, Hwang EC, Kang TW, Kwon DD, et al. Preoperative hydronephrosis and diabetes mellitus predict poor prognosis in upper urinary tract urothelial carcinoma. Can Urol Assoc J. 2013;7:E215-20.

8. Zhang X, Zhu Z, Zhong S, Xu T, Shen Z. Ureteral tumours showing a worse prognosis than renal pelvis tumours may be attributed to ureteral tumours more likely to have hydronephrosis and less likely to have haematuria. World J Urol. 2013;31:155-60.

9. Rajcani J, Kajo K, Adamkov M, Moravekova E, Lauko L, Felcanova D, et al. Immunohistochemical characterization of urothelial carcinoma. Bratisl Lek Listy. 2013;114:431-8.

10. Mazzucchelli R, Scarpelli M, Galosi AB, Di Primio R, LopezBeltran A, Cheng L, et al. Pathology of upper tract urothelial carcinoma with emphasis on staging. Int J Immunopathol Pharmacol. 2014;27:509-16.

11. Xu S, Gu G, Ni Q, Li N, Yu K, Li X, et al. The expression of AEG-1 and cyclin D1 in human bladder urothelial carcinoma and their clinicopathological significance. Int J Clin Exp Med. 2015;8:21222-8.

12. Mann SA, Lopez-Beltran A, Massari F, Pili R, Fiorentino M, Koch MO, et al. Targeting the programmed cell death-1 path- 
way in genitourinary tumors: current progress and future perspectives. Curr Drug Metab. 2017;18:700-11.

13. Bellmunt J, Mullane SA, Werner L, Fay AP, Callea M, Leow JJ, et al. Association of PD-L1 expression on tumor-infiltrating mononuclear cells and overall survival in patients with urothelial carcinoma. Ann Oncol. 2015;26:812-7.

14. Adachi K, Tamada K. Immune checkpoint blockade opens an avenue of cancer immunotherapy with a potent clinical efficacy. Cancer Sci. 2015;106:945-50.

15. Reis ST, Leite KR, Mosconi Neto A, Pontes Junior J, Viana NI, Antunes AA, et al. Immune expression of E-cadherin and alpha, beta and gamma-catenin adhesion molecules and prognosis for upper urinary tract urothelial carcinomas. Int Braz J Urol. 2012;38:466-73.

16. Cho J, Ha SY, Kim SH, Sung HH, Kwon GY. Prognostic significance of epithelial-mesenchymal transition phenotypes in upper urinary tract urothelial carcinoma. Pathol Res Pract. 2018;214:547-54.

17. Romkes M, Chern HD, Lesnick TG, Becich MJ, Persad R, Smith $\mathrm{P}$, et al. Association of low CYP3A activity with p53 mutation and CYP2D6 activity with Rb mutation in human bladder cancer. Carcinogenesis. 1996;17:1057-62.

18. Lonn U, Lonn S, Friberg S, Nilsson B, Silfversward C, Stenkvist B. Prognostic value of amplification of c-erb-B2 in bladder carcinoma. Clin Cancer Res. 1995;1:1189-94.

19. Castillo-Martin M, Domingo-Domenech J, Karni-Schmidt O, Matos T, Cordon-Cardo C. Molecular pathways of urothelial development and bladder tumorigenesis. Urol Oncol. 2010;28: 401-8.

20. Cordon-Cardo C. Molecular alterations associated with bladder cancer initiation and progression. Scand J Urol Nephrol Suppl. 2008:154-65.

21. Korkolopoulou P, Christodoulou P, Kapralos P, Exarchakos M, Bisbiroula A, Hadjiyannakis M, et al. The role of p53, MDM2 and c-erb B-2 oncoproteins, epidermal growth factor receptor and proliferation markers in the prognosis of urinary bladder cancer. Pathol Res Pract. 1997;193:767-75.

22. Vollmer RT, Humphrey PA, Swanson PE, Wick MR, Hudson ML. Invasion of the bladder by transitional cell carcinoma: its relation to histologic grade and expression of p53, MIB-1, c-erb B-2, epidermal growth factor receptor, and bcl-2. Cancer. 1998;82:715-23.

23. Fontana LO, Garcia Garcia F, Arcas Martinez Salas I, Garcia Ligero J, Tomas Ros M, Rico Galiano JL, et al. The expression of p53 and c-erb-2 in transitional cell carcinoma of the kidney pelvis and ureter and its relation to tumor progression and survival. Arch Esp Urol. 2002;55:792-6.

24. Kunju LP, Mehra R, Snyder M, Shah RB. Prostate-specific antigen, high-molecular-weight cytokeratin (clone 34betaE12), and / or p63: an optimal immunohistochemical panel to distinguish poorly differentiated prostate adenocarcinoma from urothelial carcinoma. Am J Clin Pathol. 2006;125:675-81.

25. Oya M, Schmidt B, Schmitz-Drager BJ, Schulz WA. Expression of G1-->S transition regulatory molecules in human urothelial cancer. Jpn J Cancer Res. 1998;89:719-26.

26. El-Gendi S, Abu-Sheasha G. Ki-67 and cell cycle regulators p53, p63 and cyclinD1 as prognostic markers for recurrence/ progression of bladder urothelial carcinoma. Pathol Oncol Res. 2018;24:309-22.

27. Amin MB, Trpkov K, Lopez-Beltran A, Grignon D; Members of the ISUP Immunohistochemistry in Diagnostic Urologic Pathology Group. Best practices recommendations in the application of immunohistochemistry in the bladder lesions: report from the International Society of Urologic Pathology consensus conference. Am J Surg Pathol. 2014;38:e20-34.

28. Contal C, O'Quigley J. An application of checkpoint methods in studying the effect of age on survival in breast cancer. Comput Stat Data Anal. 1999;30:253-70.

29. Connolly SS, Rochester MA; BAUS. Nephroureterectomy surgery in the UK in 2012: British Association of Urological Surgeons (BAUS) Registry data. BJU Int. 2015;116:780-90. 